\title{
Commentary
}

\section{Alveolar Soft Part Sarcoma}

\section{Are We at the End or Just the Beginning of Our Quest?}

\author{
Sharon W. Weiss \\ From the Department of Pathology and Laboratory Medicine, \\ Emory University School of Medicine, Atlanta, Georgia
}

Exactly 50 years ago this year the seminal paper on alveolar soft part sarcoma (ASPS) appeared in the journal Cancer. ${ }^{1}$ Drawing on the archives of Memorial Sloan Kettering Cancer Center, Christopherson et al ${ }^{1}$ described 12 patients, mostly young individuals, with a distinctive sarcoma composed of organoid nests of large eosinophilic cells. The loss of central cohesion within the nests created an alveolar pattern that figured prominently into the authors' choice of names. These rare tumors with a predilection for the deep soft tissues of the extremities are characterized by an indolent, but inexorably fatal, course often spanning decades. A significant number of patients present with metastasis at the time of presentation, denoting early dissemination. ${ }^{2}$ Several years after this publication, the distinctive periodic acid-Schiff (PAS) positive-diastase resistant rhomboid to polygonal crystals (and their precursor granules) which characterize this lesion, were noted. ${ }^{3}$ Consisting of membrane-bounded parallel arrays of rigid fibers with a diameter of 4 to $6 \mathrm{~nm}$ and a periodicity of $10 \mathrm{~nm}$, these structures are a characteristic, if not pathognomonic, feature of this tumor which has fascinated pathologists for decades. ${ }^{4}$ While serving an important diagnostic role in separating this tumor from others which it may resemble (eg, renal cell carcinoma, paraganglioma), these crystalline structures have also tantalized us with the promise that if only we could identify their contents, we might discover the histogenesis of this tumor.

For 50 years we have been on a quest to unravel the contents of these crystals. Like many quests we have made some false starts, taken some unusual turns, and learned some important lessons in the process. The popular use of immunohistochemistry beginning in the late 1970s allowed us for the first time to turn attention to these crystals by applying a host of antibodies to these tumors with variable and sometimes conflicting results. $^{5-13}$ The most celebrated of all theories regarding the alveolar soft part sarcoma was that it represented an unusual myogenous tumor closely related to a rhabdo- myosarcoma. ${ }^{9,10}$ Numerous immunohistochemical studies, however, demonstrated muscle markers in less than half of ASPS. ${ }^{5}$ Actin was identified in about 20 to $30 \%$ of cases, whereas desmin, an intermediate filament associated with both smooth or skeletal muscle differentiation was expressed in nearly $40 \%$. Since desmin may be expressed in non-myogenous tumors on an aberrant basis, the ability to identify the more specific nuclear regulatory proteins, associated with skeletal muscle differentiation (ie, myoD1 and myogenin), in tissue sections seemed a more certain and expeditious way to answer this question. However, early reports citing the presence of myoD1 in this tumor were fraught with technical and interpretive problems. ${ }^{9}$ It was not until the study by Wang et al, ${ }^{11}$ using immunohistochemistry along with Western blot analysis, was it decisively shown that, whereas the tumors often expressed desmin, they did not express skeletal muscle nuclear regulatory proteins and, in all probability, did not represent a skeletal muscle tumor. Attempts to demonstrate catecholamines and related substances have also failed and therefore, effectively excluded the possibility that these tumors were a variant of paraganglioma. ${ }^{12}$ Perhaps the most whimsical and short-lived of all theories was the notion that alveolar soft part sarcomas were extra-renal renin-producing tumors (so-called malignant angioreninomas) based on immunoreactivity of the granules to renin antiserum. ${ }^{13}$ Other studies failed to corroborate these findings, ${ }^{7}$ and, by way of passing, hypertension has never been reported as a paraneoplastic symptom in with ASPS. As a result of the myriad of immunohistochemical articles over the years relating to this tumor, hopefully we have learned to be cautious in accepting novel findings particularly when they relate to new antibodies that have not been extensively used or performance tested.

Despite this cautionary note, the paper in this issue by Ladanyi et $\mathrm{al}^{14}$ seems to represent the long-awaited unveiling of the identity of these granules. It is gratifying that

Accepted for publication January 4, 2002.

Address reprint requests to Sharon W. Weiss, Department of Pathology and Laboratory Medicine, Emory University Hospital, 1364 Clifton Road, Atlanta, GA 30322. E-mail: sharon_weiss@emory.org. 
this study also emanates from the institution that 50 years ago began the scientific dialogue about this enigmatic tumor. As is often the case with novel observations, the authors discovered the identity of the granules serendipitously in the course of characterizing a polyclonal antibody to the monocarboxylate transporter 1 (MCT1) in a variety of tissues and tumors. MCT1, one of a family of transporter proteins which catalyzes the rapid transport of monocarboxylates such as lactate across plasma membranes, is located ubiquitously in all tissues but is especially prevalent in cardiac and skeletal muscle and seems to roughly correlate with the abundance of mitochondria. ${ }^{15}$ Normally the protein is associated with the rough endoplasmic reticulum and is transported to the plasma membrane in association with its chaperone CD147 where it can be detected as surface staining. Important interactions between CD147 and MCT1 also occur in the plasma membrane. As is elegantly shown in the current article by Ladanyi et al, ${ }^{14}$ alveolar soft part sarcomas contain an abundance of MCT1 not only on the surface of the cells but also within the cytoplasm and in the region of the characteristic crystals. Immunolocalization of CD147 was observed in pattern similar to MCT1. The authors have avoided the traps of earlier studies by validating their immunohistochemical findings through Western blot analysis for the protein. Moreover, comparison of serial histochemical and immunohistochemical stains for the crystals confirmed congruence between the PAS positive crystalline material and the immunoreactivity for MCT1. Finally, using ultrastructural immunohistochemistry, MCT1 and CD147 were localized to cytoplasmic crystals, their precursor granules, and mitochondria. Thus substantial evidence is presented that the crystals of ASPS contain crystallized complexes of both proteins. Obviously, their findings do not exclude the possibility that other substances may be present in the granules as well.

To return to our earlier question, however, does the identity of the crystals help us to understand the histogenesis (or line of differentiation) of the tumor? The immediate answer to the question is no. MCT1 is a ubiquitously expressed protein involved in basic metabolic processes in a variety of cells, although its predilection for skeletal and cardiac muscle might make us pause to again reconsider a myogenic tumor. CD147 is a broadly expressed plasma membrane glycoprotein which not only serves as role as a chaperone for MCT1, but also interacts with the latter at the level of the plasma membrane suggesting a regulatory function. ${ }^{16-18}$ Another intriguing feature of CD147 is that it is also induces the production of matrix metalloproteinases by fibroblasts ${ }^{19}$ and its overexpression by some cancer cells has been associated with increased tumorigenic potential in nude mice. ${ }^{19}$ One could, therefore, envision how a tumor with increased expression of CD147 could be associated with early invasion, vascular permeation, and metastasis, as characteristically occurs in ASPS. In fact, ASPS may be the soft tissue sarcoma in which vascular invasion is most prevalent even in early stage disease. Perhaps also, as suggested by the authors, even the characteristic alveolar pattern may be explained by matrix degradation as a result of metalloproteinase production. Although the histogenesis of the ASPS still eludes us, we may have discovered deeper biological truths about the tumor.

Of course the most intriguing question is how the recently described translocation in ASPS relates to the accumulation of crystalline deposits of MCT1 and CD147. One might logically assume that there is a relationship given the fundamental role that fusion genes and their derivative proteins play in soft tissue sarcomas in general. This translocation, resulting in $\operatorname{der} 17 t(X ; 17)(p 11.2$; q25), fuses the TFE3 transcription factor gene at Xp11 to a novel gene at 17q25 designated APSL. ${ }^{20}$ Although the translocation in ASPS differs from that in most sarcomas in that it is unbalanced, it shares a common theme in that one partner represents a transcription factor, suggesting transcriptional deregulation as a mechanism for tumorigenesis. The most obvious and intellectually satisfying explanation of the relationship between the translocation and the crystals would be that the promotor of either MCT1 or CD147 is activated by TFE3 resulting in overproduction of the proteins. Although the MCT1 promoter is unknown, the CD147 promoter could potentially be activated by TFE3 given the sequence data. We await further information on this point. There are, of course, other explanations for the accumulation of these protein products, as suggested by the authors, such as impaired trafficking to their target destination.

Thus the paper by Ladanyi et $\mathrm{al}^{14}$ in this issue of The American Journal of Pathology has revealed (at least partially) the identity of the enigmatic crystals of ASPS and at the same time given us some plausible mechanisms for the way in which the fusion oncogene may relate to the crystals. One thing we have not learned, however, is the histogenesis (or line of differentiation) of ASPS. Implied in this question is our naive belief that all tumors must necessarily relate to some normal tissue type. In many sarcomas this is true. For example, the myxoid liposarcoma recapitulates embryonic fat and its characteristic translocation results in deregulation of normal adipocytic differentiation. Not all sarcomas may follow that paradigm and the ASPS may be the prime example. Some sarcomas may express a phenotype corresponding to no known normal tissue or a "scrambled" phenotype of several tissue types. Hopefully, we will not be disappointed, after learning the contents of the enigmatic crystals, to realize that ASPS is still a lesion of "uncertain histogenesis." In the next half century, perhaps we should decide that it is time to rephrase the question.

\section{References}

1. Christopherson WM, Foote FW, Stewart FW: Alveolar soft part sarcomas: structurally characteristic tumors of uncertain histogenesis. Cancer 1952, 5:100-111

2. Lieberman PH, Brennan MF, Kimmel M, Erlandson RA, Garin-Chesna $P$, Fiehinger BY: Alveolar soft part sarcoma: a clinicopathologic study of half a century. Cancer 1989, 63:1-13

3. Masson P: Tumeurs Humaines: Histologie, Diagnostics, et Techniques, ed 2. Paris, Librairie Maloine, 1959

4. Shipkey FH, Lieberman PH, Foote FW, Stewart FW: Ultrastructure of alveolar soft part sarcoma. Cancer 1964, 17:821-830

5. Weiss SW, Goldblum JR: Malignant tumors of uncertain type. Enz- 
inger and Weiss's Soft Tissue Tumors, ed 4. Philadelphia, MosbyHarcourt, 2001, pp 1509-1521

6. Mukai M, Torikata C, Shimoda T, Iri H: Alveolar soft part sarcoma: assessment of immunohistochemical demonstration of desmin using paraffin sections and frozen sections. Virchows Arch Pathol Anat 1989, 414:503-509

7. Mukai M, Iri H: Nakajima T, Hirose S, Torikata C, Kageyama K, Ueno $\mathrm{M}$, Morikami K: Alveolar soft part sarcoma: a review of its histogenesis and further studies based on electron microscopy, immunohistochemistry, and biochemistry. Am J Surg Pathol 1983, 7:679-689

8. Ordonez NG, Ro JY, Mackay B: Alveolar soft part sarcoma: an ultrastructural and immunocytochemical investigation of its histogenesis. Cancer 1989, 63:1721-1736

9. Rosai J, Dias P, Parham DM, Shapiro DN, Houghton P: myoD1 protein expression in alveolar soft part sarcoma as confirmatory evidence of its skeletal muscle differentiation: Am J Surg Pathol 1991, 15:974-981

10. Foschini MP, Eusebi V: Alveolar soft part sarcoma: a new type of rhabdomyosarcoma? Semin Diagn Pathol 1994, 11:58-68

11. Wang NP, Bacchi, Ce, Jiang JJ, McNutt MA, Gown AM: Does alveolar soft part sarcoma exhibit skeletal muscle differentiation? An immunohistochemical and biochemical study of myogenic regulatory protein expression. Mod Pathol 1996, 9:496-506

12. Auerbach HE, Brooks JJ: Alveolar soft part sarcoma: a clinicopathologic and immunohistochemical study. Cancer 1987, 60:66-73

13. DeSchryver-Kecskemeti K, Kraus FT, Engleman BA: Alveolar softpart sarcomas: a malignant angioreninoma; histochemical, immuno- cytochemical, and electron-microscopic study of four cases. Am J Surg Pathol 1982, 6:5-18

14. Ladanyi M, Antonescu CR, Drobnjak M, Baran A, Lui MY, Golde DW, Cordon-Cardo C: The precrystalline cytoplasmic granules of alveolar soft part sarcoma contain monocarboxylate transporter 1 and CD 147. Am J Pathol, 2002, 160:1215-1221

15. Halestrap AP, Price NT: The proton-linked monocarboxylate transporter (MCT) family: structure, function, and regulation. Biochem $\mathrm{J}$ 1999, 343:281-299

16. Kirk P, Wilson MC, Heddle C, Brown MH, Barclay AN, Halestrap AP CD147 is tightly associated with lactate transporters MCT1 and MCT4 and facilitates their cell surface expression. EMBO J 2000, 19:38963904

17. Sun J, Hemler ME: Regulation of MMP-1 and MMP-2 production through CD147/extracellular matrix metalloproteinase inducer interactions. Cancer Res 2001, 61:2276-2281

18. Guo H, Majmudar G, Jensen TC, Biswas C, Toole BP, Gordon MK: Characterization of the gene for human EMMPRIN, a tumor cell surface inducer of matrix metalloproteinases. Gene 1998, 220:99-108

19. Zucker S, Hymowitz, Rollo EE, Mann R, Conner CE, Cao J, Foda HD, Tompkins DC, Toole BP: Tumorigenic potential of extracellular matrix metalloproteinase inducer. Am J Pathol 2001, 158:1921-1928

20. Ladanyi M, Lui MY, Antonescu CR, Krause-Boehm A, Meindl A, Argani $P$, Healey $J H$, et al: The $\operatorname{der}(17) t(X ; 17)(p 11 ; q 25)$ of human alveolar soft part sarcoma fuses the TFE transcription factor gene to ASPL, a novel gene at 17q25. Oncogene 2001, 20:48-57 